\title{
PIPERIDINE MEDIATED SYNTHESIS OF PRENYLATED CHALCONES AND 8-SUBSTITUTED -2, 5-DIHYDRO-2-(4- TOLYBENZO)-5-(3-METHYLBUT-2-ENYLOXY) PHENOL-1, 5- BENZOTHIAZEPINES AND ITS DERIVATIVES AS ANTI CANCER AGENTS
}

\author{
M. Sudha Rani ${ }^{1,2}$, N. Ch. Kalyani ${ }^{1}$, Ch. Murthy ${ }^{2}$, N. Bhasker ${ }^{1,2,{ }^{*}}$ \\ and B.V. Subba Reddy ${ }^{3}$ \\ ${ }^{1}$ Division of Chemistry, Department of Sciences and Humanities, Guru Nanak Institution of \\ Technical Campus (JNTU), Hyderabad-501 506, Telangana, India. \\ ${ }^{2}$ Division of Chemistry, Department of Sciences and Humanities, Vegans' Foundation for \\ Science, Technology and Research University (Vignan University), \\ Vadlamudi-522 213, Guntur, Andhra Pradesh, \\ ${ }^{3}$ Indian Institute of Chemical Technology, Hyderabad-505 001, Telangana, India. \\ *E-mail: nbhasker28@gmail.com
}

\begin{abstract}
1, 5-benzothiazepine is the main seven-membered heterocyclic ring system and having several cardiac, psychotherapeutic activities. Which has been synthesized by a catalytic amount of piperidine mediated condensation of dry toluene with 5-substituted-2-Amino benzenethiols (2) and Prenyloxy chalcones (3). The corresponding prenyloxy chalcones (3) were synthesized by piperidine mediated claisen-Schmidt condensation of an ethanolic solution of 4-prenyloxy 2-hydroxy acetophenone (1) with aromatic aldehydes. It was planned to use a weaker base like piperidine instead of using a strong base to enhance the better yields. The structures have been established on the basis of elemental (C, H, N) analysis, IR, ${ }^{1} \mathrm{H}$ NMR, Mass spectral data. The compounds (3) and (5) were screened for antimicrobial activities against a variety of bacterial agent. The anti-oxidative activities were also determined.

Keywords: 8-Substituted-2, 5-Dihydro-2-(4-tolybenzo)-5-(3-methybut-2-enyloxy) phenol-1, 5 Benzothiazepines, Benzothiazepines Derivatives, Prinyloxy Chalcones, Anti Cancer Activity
\end{abstract}

(C) RASĀYAN. All rights reserved

\section{INTRODUCTION}

1,5-Benzothiazepine and 1,5-benzodiazipine are the two main seven-membered heterocyclic ring systems reported for their cardiac and psychotherapeutic activities. The successful introduction of diltiazem and clentiazem for angina pectoris, hypertension, arrhythmias and other related cardiac disorders proved potential of the 1,5-benzothiazepine moiety. Subsequently 1, 5-benzodiazepines were highlighted as important biologically active scaffolds. Also, the discovery of thiazesim and quetiapine fumarate as psychotropic agents attracted much attention worldwide. 1, 5-Benzothiazepines having a different heterocyclic group at different positions having shown antiulcer ${ }^{1,2}$, analgesic, ${ }^{3}$ vasodepressant, ${ }^{4}$ antihypertensive, ${ }^{5}$ anti-amnesia and antidementia, ${ }^{6}$ antibacterial and antifungal, ${ }^{7}$ and insecticidal, ${ }^{8}$ activity. 1, 5-Benzothiazepines having a heterocyclic group at the different position of the ring have been found to be of psychopharmacological use. Various other useful properties ${ }^{9-20}$ have been shown by 1,5 Benzothiazepines and different compounds having heterocyclic function have been synthesized. The biodynamic nature of 1,5-benzothiazepine derivatives led to the current synthesis of 1,5-benzothiazepines having various substituents at positions 2,4 and 8 , which may prove to be medicinally potent. In this

Rasayan J. Chem., 12(2), 796-802(2019)

http://dx.doi.org/10.31788/RJC.2019.1224078

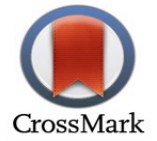


quest. The reactions of 5-substituted-2-aminobenzenethiols with compounds having $\alpha, \beta$-unsaturation in conjugation with carbonyl system in acidic, basic and neutral media to give 2, 4-diaryl-2, 5-dihydro-1, 5benzo-thiazepines, ${ }^{21}$ 2-carboxy-2, 3-dihydro-4-aryl-1,5-benzothiazepines, ${ }^{22}$ 2,5-dihydro-2-(4-pyridyl)-4(2-thienyl)-1,5-benzothiazepines ${ }^{23}$ and tetra cyclic benzopyranobenzo thiazepines ${ }^{24}$ have been reported. Herein is reported the synthesis of having various substituents at positions 2,4 and 8 . We mainly cover structural elucidation of newly synthesized compounds done along with the brief description of the targets and report piperidine mediated synthesis. All the compounds have been tested for antibacterial activity. It was planned to use a weaker base like piperidine instead of using a strong base to enhance the better yields.

\section{EXPERIMENTAL}

Melting points were determined in open capillary tubes and were not corrected. IR spectra $\left(\mathrm{KBr}, \lambda{ }_{\max }\right.$ in $\mathrm{cm}^{-1}$ ) were recorded on a Bruker IFS 66V spectrometer, ${ }^{1} \mathrm{H}$ NMR spectra (chemical shifts in $\delta$, Ppm) on a Gemini-400 MHz spectrometer in $\mathrm{CDCl}_{3}$ using tetramethylsilane as the internal standard and MS spectra on a VG $7070 \mathrm{H}$ spectrometer. The purity of the compounds was verified by TLC (benzene/ethyl acetate, 9:1), using Merck brand Silica Gel-G plates and spotting was done using iodine.

\section{4-Prenyloxy 2-hydroxy $\beta$-resaacetophenone, 1}

A solution of $\beta$-resacetophenone $(0.5 \mathrm{~g})$ in acetone $(10 \mathrm{ml})$ was refluxed with prenyl bromide $(0.4 \mathrm{ml})$ and anhydrous potassium carbonate $(2 \mathrm{gms})$ for $3 \mathrm{hrs}$. The product crystallized from light petroleum ether at low temperature as colorless thick needles $(0.5 \mathrm{gms})$, m.p. $45-47^{\circ} \mathrm{C}$, red ferric reaction; $\mathrm{R}_{\mathrm{F}} 0.30$, solvent (benzene - light petroleum 1:1); $\mathrm{V}_{\max } 1640 \mathrm{Cm}^{-1}$.

\section{General procedure for the synthesis of 4-prenyloxy 2-hydroxy chalcones, 3}

To a mixture of 4-prenyloxy 2-hydroxyacetophenone ( $0.01 \mathrm{~mole})$ and aromatic aldehyde $(0.01 \mathrm{~mole})$ were dissolved in EtOH $(50 \mathrm{~mL})$ and Piperidine $(1 \mathrm{~mL})$ was added and refluxed. After the completion of the reaction, which was monitored by TLC, ethanol was distilled off and the residue was poured on ice water $(100 \mathrm{~mL})$. It was kept overnight in the refrigerator. The resulting solid was collected by filtration, washed with distilled water and crystallized from methanol to give corresponding chalcones 3a-d.

\section{Compound 3a}

Dirty Yellow solid, mp 87-88 ${ }^{\circ} \mathrm{C}$. IR $\left(\mathrm{KBr}, \mathrm{cm}^{-1}\right): 1646\left(v_{\mathrm{C}=\mathrm{O}}\right), 1625\left(v_{\mathrm{CH}=\mathrm{CH}}\right):{ }^{1} \mathrm{H}$ NMR $\left(\mathrm{CDCl}_{3}, 400\right.$ MHz): $7.92\left(\mathrm{~d}, 1 \mathrm{H}, \mathrm{C}_{\alpha} \mathrm{H}, J=15.3 \mathrm{~Hz}\right), 8.12\left(\mathrm{~d}, 1 \mathrm{H}, \mathrm{C}_{\beta} \mathrm{H}, J=15.3 \mathrm{~Hz}\right), 7.23-7.56(\mathrm{~m}, 6 \mathrm{H}) . \mathrm{MS}(\mathrm{m} / \mathrm{z}, \%)$ : $204\left(\mathrm{M}^{+}, 100\right), 188$ (34), 176 (27), 172 (52), 112 (13), 93 (12). Anal. Calcd. for $\mathrm{C}_{11} \mathrm{H}_{8} \mathrm{O}_{2}$ S: C, 64.52; $\mathrm{H}$, 3.86; O, 15.50. Found: C, 64.71; H, 3.95; O, 15.68 .

\section{Compound 3b}

Yellow solid, mp 91-92 ${ }^{\circ} \mathrm{C}$. IR $\left(\mathrm{KBr}, \mathrm{cm}^{-1}\right): 1650\left(v_{\mathrm{C}=\mathrm{O}}\right), 1630\left(v_{\mathrm{CH}=\mathrm{CH}}\right) ;{ }^{1} \mathrm{H}$ NMR $\left(\mathrm{CDCl}_{3}, 400 \mathrm{MHz}\right) ; 6.92$ $\left(\mathrm{d}, 1 \mathrm{H}, \mathrm{C}_{\alpha} \mathrm{H}, J=15.3 \mathrm{~Hz}\right), 7.82\left(\mathrm{~d}, 1 \mathrm{H}, \mathrm{C}_{\beta} \mathrm{H}, J=15.3 \mathrm{~Hz}\right), 7.13-7.26(\mathrm{~m}, 6 \mathrm{H}): . \mathrm{MS}(\mathrm{m} / \mathrm{z}, \%): 220\left(\mathrm{M}^{+}\right)$; $220\left(\mathrm{M}^{+}, 100\right) 203$ (37), 188 (72), 110 (28), 109 (42), 93 (12), 84 (14), 30 (18), 28 (15). Anal. Calcd. for $\mathrm{C}_{11} \mathrm{H}_{8} \mathrm{O} \mathrm{S}_{2}$ : C, 59.82; H, 3.54; O, 7.21. Found: C, 59.97; H, 3.66; O, 7.26.

\section{Compound 3c}

Light yellow solid, mp 185-186 ${ }^{\circ} \mathrm{C}$. IR $\left(\mathrm{KBr}, \mathrm{cm}^{-1}\right): 1646\left(v_{\mathrm{C}=\mathrm{O}}\right), 1625\left(v_{\mathrm{CH}=\mathrm{CH}}\right):{ }^{1} \mathrm{H}$ NMR $\left(\mathrm{CDCl}_{3}, 400\right.$ $\mathrm{MHz}) ; 6.82\left(\mathrm{~d}, 1 \mathrm{H}, \mathrm{C}_{\alpha} \mathrm{H}, J=15.3 \mathrm{~Hz}\right), 7.64\left(\mathrm{~d}, 1 \mathrm{H}, \mathrm{C}_{\beta} \mathrm{H}, J=15.3 \mathrm{~Hz}\right), 7.03-7.29(\mathrm{~m}, 6 \mathrm{H},) . \mathrm{MS}(\mathrm{m} / \mathrm{z}, \%)$ : $204\left(\mathrm{M}^{+}, 88\right), 188$ (100), 176 (36), 175 (27), 173 (13), 112 (11), 94 (22), 72 (8), 67 (48), 17 (10), 14 (12). Anal. Calcd for $\mathrm{C}_{11} \mathrm{H}_{8} \mathrm{O}_{2} \mathrm{~S} ; \mathrm{C}, 64.81 ; \mathrm{H}, 3.82 ; \mathrm{O}, 15.64$. Found: C, 64.89; H, 3.95; O, 15.68.

\section{Compound 3d}

Dork Yellow solid, mp 95-96 ${ }^{\circ} \mathrm{C}$. IR $\left(\mathrm{KBr}, \mathrm{cm}^{-1}\right): 1648\left(v_{\mathrm{C}=\mathrm{O}}\right), 1627\left(v_{\mathrm{CH}=\mathrm{CH}}\right) ;{ }^{1} \mathrm{H}$ NMR $\left(\mathrm{CDCl}_{3}, 400\right.$ $\mathrm{MHz}) ; 6.92\left(\mathrm{~d}, 1 \mathrm{H}, \mathrm{C}_{\alpha} \mathrm{H}, J=15.3 \mathrm{~Hz}\right), 7.82\left(\mathrm{~d}, 1 \mathrm{H}, \mathrm{C}_{\beta} \mathrm{H}, J=15.3 \mathrm{~Hz}\right), 7.13-7.26(\mathrm{~m}, 6 \mathrm{H}) . \mathrm{MS}(\mathrm{m} / \mathrm{z}, \%)$ 
RASĀYAN J. Chem.

Vol. 12 | No. 2 |796-802| April - June | 2019

$188\left(\mathrm{M}^{+}, 100\right), 172$ (36), 112 (52), 88 (23), 64 (56), 30 (12), 18 (10). Anal. Calcd. for $\mathrm{C}_{11} \mathrm{H}_{8} \mathrm{O}_{3}: \mathrm{C}, 70.20$; H, 4.25; O, 25.46. Found: C, 70.21; H, 4.29; O, 25.51;

General Procedure for Synthesis of Prenyloxy, 5-Substituted-1, 5-Benzothiazepines, 5

5-substituted-2-Amino-benzenethiol $4(0.001 \mathrm{~mol})$ and prenyloxy chalcone $\mathbf{3}(0.001 \mathrm{~mol})$ was refluxed in dry toluene containing a catalytic amount of piperidine $(1 \mathrm{~mL})$ for $7 \mathrm{hr}$. The crude solid obtained on removal of solvent gave a solid, which on purification by recrystallization from dry methanol gave 1, 5benzothiazepine derivative 5. All Compounds were prepared by using similar procedures. However, the completion of the reaction in case of $\mathbf{5 c}, \mathbf{5 h}$ required $8 \mathrm{hr}$ and $\mathbf{5 b}, \mathbf{5 e}$ and $\mathbf{5 f}$ required $6 \mathrm{hr}$ heating with reflux. The total spectral data, physical data and analytical data of newly synthesized compounds have been given

\section{Compound 5a}

Yellow solid, mp 92-94 ${ }^{\circ} \mathrm{C}$. IR $\left(\mathrm{KBr}, \mathrm{cm}^{-1}\right)$ : $1608\left(\mathrm{~V}_{\mathrm{N}=\mathrm{C}}\right) ;{ }^{1} \mathrm{H}$ NMR $\left(\mathrm{CDCl}_{3}, 400 \mathrm{MHz}\right): 3.83(\mathrm{~s}, 3 \mathrm{H},-$ $\left.\mathrm{OCH}_{3}\right), 4.12$ (br, $\left.1 \mathrm{H},-\mathrm{NH}\right), 6.84$ (d, $\left.1 \mathrm{H}, J=8 \mathrm{~Hz}, \mathrm{C}-2-\mathrm{H}\right), 6.92$ (d, 1H, $\left.J=8 \mathrm{~Hz}, \mathrm{C}-3-\mathrm{H}\right), 6.44$ (s, $1 \mathrm{H}, \mathrm{C}_{9^{-}}$ $\mathrm{H}), 6.82-7.85(\mathrm{~m}, 9 \mathrm{H}) . \mathrm{MS}(\mathrm{m} / \mathrm{z}, \%): 341\left(\mathrm{M}^{+}, 67\right), 343\left(\mathrm{M}+2^{+}, 48\right), 310$ (42), 274 (22), 258 (100), 243 (16), 227 (9), 154 (23), 109 (36), 83 (10), 80 (32), 67 (89), 31 (10). Anal. Calcd for $\mathrm{C}_{18} \mathrm{H}_{15} \mathrm{O}_{2} \mathrm{~S}_{2} \mathrm{~N}$ (341): C 63.34; H, 4.43; N, 4.10; O, 9.37. Found: C, 63.45; H, 4.55; N, 4.12; O, 9.39.

\section{Compound 5b}

Yellow solid, mp 97-98 ${ }^{\circ} \mathrm{C}$. IR $\left(\mathrm{KBr}, \mathrm{cm}^{-1}\right): 1605\left(\mathrm{~V}_{\mathrm{N}=\mathrm{C}}\right) .{ }^{1} \mathrm{H}$ NMR $\left(\mathrm{CDCl}_{3}, 400 \mathrm{MHz}\right) ; 2.41(\mathrm{~s}, 3 \mathrm{H}), 4.00$ (br, 1H), $6.86(\mathrm{~d}, 1 \mathrm{H}, J=8 \mathrm{~Hz}), 6.91(\mathrm{~d}, 1 \mathrm{H}, J=8 \mathrm{~Hz}), 6.36\left(\mathrm{~s}, 1 \mathrm{H}, \mathrm{C}_{9}-\mathrm{H}\right), 6.82-7.91(\mathrm{~m}, 9 \mathrm{H}) . \mathrm{MS}(\mathrm{m} / \mathrm{z}$, \%): $325\left(\mathrm{M}^{+}, 50\right), 310$ (58), 258 (100), 253 (42), 201 (16), 156 (9), 154 (23), 109 (36), 89 (18), 82 (23), 67 (46), 28 (10).

Anal. Calcd. for $\mathrm{C}_{18} \mathrm{H}_{15} \mathrm{O} \mathrm{S}_{2} \mathrm{~N}$ : C 66.43; H, 4.65; N, 4.30; O, 4.92, S, 19.71. Found: C, 66.55; H, 4.73; N, 4.42; O, 5.03; S, 19.82;

\section{Compound 5c}

Yellow solid, mp 85-87 ${ }^{\circ} \mathrm{C}$. IR $\left(\mathrm{KBr}, \mathrm{cm}^{-1}\right)$ : $1605\left(\mathrm{~V}_{\mathrm{C}=\mathrm{N}}\right) .{ }^{1} \mathrm{H}$ NMR $\left(\mathrm{CDCl}_{3}, 400 \mathrm{MHz}\right.$,) 3.83 (s, 3H), 4.12 (br, 1H), 6.84 (d, 1H, J = 8 Hz), 6.92 (d, 1H, J = $8 \mathrm{~Hz}), 6.42$ (s, 1H, C9-H), 6.82-8.85 (m, 9H). MS (m/z, \%): $357\left(\mathrm{M}^{+}, 63\right), 343$ (48), 326 (100) 310 (22), 290 (12), 284 (32), 240 (16), 225 (9), 152 (23), 109 (36), 83 (10), 80 (32), 47 (89), 27 (10). Anal. Calcd. for $\mathrm{C}_{18} \mathrm{H}_{15} \mathrm{O} \mathrm{S}_{3} \mathrm{~N}$ : C, 60.47; H, 4.23; N, 3.97; O, 4.48; S, 26.91. Found: C, 60.55; H, 4.33; N, 4.02; O, 4.57; S, 27.05.

\section{Compound 5d}

Bright yellow solid, mp 95-96 ${ }^{\circ} \mathrm{C}$. IR $\left(\mathrm{KBr}, \mathrm{cm}^{-1}\right)$ : $1608\left(\mathrm{~V}_{\mathrm{N}=\mathrm{C}}\right) .{ }^{1} \mathrm{H}$ NMR $\left(\mathrm{CDCl}_{3}, 400 \mathrm{MHz}\right): 2.43$ (s, 3H), 4.12 (br, 1H), 6.84 (d, 1H, J = 8 Hz), 6.92 (d, 1H, J = $8 \mathrm{~Hz}), 6.48$ (s, 1H, C9-H), 6.82-8.85 (m, 9H). MS (m/z, \%): $341\left(\mathrm{M}^{+}, 65\right), 343\left(\mathrm{M}+2^{+}, 48\right), 326$ (100), 274 (22), 253 (89), 240 (10), 227 (9), 154 (23), 109 (36), 73 (10), 67 (32), 47 (28), 27 (23). Anal. Calcd. for $\mathrm{C}_{18} \mathrm{H}_{15} \mathrm{~S}_{3} \mathrm{~N}$ : C 63.34; H, 4.43; N, 4.10; S, 28.17. Found: C, 63.45; H, 4.52; N, 4.12; S, 28.26;

\section{Compound 5e}

Yellow solid, mp 85-86 ${ }^{\circ} \mathrm{C}$. IR $\left(\mathrm{KBr}, \mathrm{cm}^{-1}\right): 1610\left(\mathrm{~V}_{\mathrm{N}=\mathrm{C}}\right) .{ }^{1} \mathrm{H} \mathrm{NMR}\left(\mathrm{CDCl}_{3}, 400 \mathrm{MHz}\right): 3.83$ (s, 3H), 4.12 (br, 1H), $6.84(\mathrm{~d}, 1 \mathrm{H}, J=8 \mathrm{~Hz}), 6.92(\mathrm{~d}, 1 \mathrm{H}, J=8 \mathrm{~Hz}), 6.52(\mathrm{~s}, 1 \mathrm{H}, \mathrm{C} 9-\mathrm{H}), 6.82-8.85(\mathrm{~m}, 9 \mathrm{H}) . \mathrm{MS}(\mathrm{m} / \mathrm{z}$, \%): $325\left(\mathrm{M}^{+}, 45\right), 310$ (58), 258 (100), 253 (42), 201 (16), 156 (9), 154 (13), 109 (43), 89 (18), 73 (20), 67 (52), 27(16). Anal. Calcd. for $\mathrm{C}_{18} \mathrm{H}_{15} \mathrm{O}_{3} \mathrm{~S} \mathrm{~N}$ : C 66.43; H, 4.65; N, 4.30; O, 4.92, S, 19.71. Found: C, 66.50; H, 4.73; N, 4.39; O, 4.98, S, 19.86;

\section{Compound $5 f$}

Dark yellow solid, mp 89-90 ${ }^{\circ} \mathrm{C}$. IR $\left(\mathrm{KBr}, \mathrm{cm}^{-1}\right)$ : $1606\left(\left(\mathrm{~V}_{\mathrm{N}=\mathrm{C}}\right) .{ }^{1} \mathrm{H}\right.$ NMR $\left(\mathrm{CDCl}_{3}, 400 \mathrm{MHz}\right)$ : 2.40 (s, $3 \mathrm{H}), 4.12(\mathrm{br}, 1 \mathrm{H}), 6.84(\mathrm{~d}, 1 \mathrm{H}, J=8 \mathrm{~Hz}), 6.92(\mathrm{~d}, 1 \mathrm{H}, J=8 \mathrm{~Hz}), 6.32\left(\mathrm{~s}, 1 \mathrm{H}, \mathrm{C}_{9}-\mathrm{H}\right), 6.82-8.85(\mathrm{~m}, 9 \mathrm{H})$. MS (m/z, \%): 309 (M+ 56), 294 (58), 242 (100), 227 (67), 206 (40), 160 (45), 134 (16), 122 (23), 67 
(46), 48 (10). Anal. Calcd. for $\mathrm{C}_{18} \mathrm{H}_{15} \mathrm{O}_{2} \mathrm{~S} \mathrm{~N}$ : C 69.88; H, 4.85; N, 4.53; O, 10.32; S,10.36. Found: C, $69.95 ; \mathrm{H}, 4.93 ; \mathrm{N}, 4.62 ; \mathrm{O}, 10.45 ; \mathrm{S}, 10.48$.

\section{Compound 5g}

Yellow solid, mp 83-84 ${ }^{\circ} \mathrm{C}$. IR $\left(\mathrm{KBr}, \mathrm{cm}^{-1}\right): 1607$ (( $\left.\mathrm{V}_{\mathrm{N}=\mathrm{C}}\right) .{ }^{1} \mathrm{H}$ NMR $\left(\mathrm{CDCl}_{3}, 400 \mathrm{MHz}\right): 3.83(\mathrm{~s}, 3 \mathrm{H}), 4.12$ (br, $1 \mathrm{H}), 6.84(\mathrm{~d}, 1 \mathrm{H}, J=8 \mathrm{~Hz}), 6.92(\mathrm{~d}, 1 \mathrm{H}, J=8 \mathrm{~Hz}), 6.31\left(\mathrm{~s}, 1 \mathrm{H}, \mathrm{C}_{9}-\mathrm{H}\right), 6.82-8.85(\mathrm{~m}, 9 \mathrm{H}) . \mathrm{MS}(\mathrm{m} / \mathrm{z}$, \%): 341 ( $\left.\mathrm{M}^{+}, 55\right), 343$ (M+2 $\left.{ }^{+}, 48\right), 310$ (100), 254 (22), 237 (89), 170 (9), 164 (16), 109 (36), 73 (10), 67 (32), 47 (28), 27 (23). Anal. Calcd for $\mathrm{C}_{18} \mathrm{H}_{15} \mathrm{O}_{2} \mathrm{~S}_{2} \mathrm{~N}$ : C 63.32; H, 4.45; N, 4.10; O, 9.37; S, 18.71. Found: C, 63.45; H, 4.53; N, 4.42; O, 9.47; S, 18.93 .

\section{Compound 5h}

Light yellow solid, mp 93-94 ${ }^{\circ} \mathrm{C}$. IR ( $\left.\mathrm{KBr}, \mathrm{cm}^{-1}\right): 1650\left(\mathrm{~V}_{\mathrm{N}=\mathrm{C}}\right) .{ }^{1} \mathrm{H}$ NMR $\left(\mathrm{CDCl}_{3}, 400 \mathrm{MHz}\right): 2.42(\mathrm{~s}, 3 \mathrm{H})$, 4.12 (br, 1H), 6.84 (d, 1H, $J=8 \mathrm{~Hz}), 6.92(\mathrm{~d}, 1 \mathrm{H}, J=8 \mathrm{~Hz}), 6.34$ (s, 1H, C9-H), 6.82-8.85 (m, 9H). MS (m/z, \%): $325\left(\mathrm{M}^{+}, 48\right) 327\left(\mathrm{M}+2^{+}, 34\right) 310$ (100), 258 (60), 253 (22), 201 (10), 156 (12), 154 (15), 109 (29), 89 (18), 73 (20), 67 (52), 27(16). Anal. Calcd. for $\mathrm{C}_{18} \mathrm{H}_{15} \mathrm{O} \mathrm{S}_{2} \mathrm{~N}$ : C, 66.43; H, 4.65; N, 4.30; O, 4.92; S, 19.71. Found: C, 66.75; H, 4.83; N, 4.72; O, 4.98; S, 19.87.

\section{RESULTS AND DISCUSSION}

The Prenyloxy chalcones $\mathbf{3}$ were prepared by reacting 4-prenyloxy 2-hydroxy acetophenone and corresponding aldehydes in EtOH $(50 \mathrm{~mL})$ and piperidine $(1 \mathrm{~mL})$ was added refluxed. After the completion of the reaction, which was monitored by TLC, ethanol was distilled off and the residue was poured on ice water $(100 \mathrm{~mL})$. It was kept overnight in the refrigerator. The resulting solid was collected by filtration, washed with distilled water and crystallized from methanol to give corresponding penyloxy chalcones 3 .

1, 5-benzothiazepines $\mathbf{5}$ were prepared by reacting Prenyloxy chalcones $\mathbf{3}$ and freshly prepared 5substituted-2-acetylthiophene 4 in dry toluene containing piperidine. The reaction is known ${ }^{25-29}$ to be initiated by nucleophilic attack of the sulpydryl electrons, whose nucleophilicity is increased in the basic medium, ${ }^{30}$ on the $\beta$-carbon atom of the 2 -propenone to give the cyclized product. Through the formation of Michael adduct intermediate, in a single step. The structures of the final products were ascertained by microanalysis for $\mathrm{C}, \mathrm{H}, \mathrm{N}$ and spectral studies comprising IR, ${ }^{1} \mathrm{H}$ NMR and MS all compounds were screened antibacterial activities. In the IR spectrum of $\mathbf{3}$ Strong absorptions for $\mathrm{C}=\mathrm{O}$ and vinylic $\mathrm{C}=\mathrm{C}$ were observed at 1646 and $1625 \mathrm{~cm}^{-1}$, respectively. The position of the vinylic $\mathrm{C}=\mathrm{C}$ appearing at a frequency lower than for an isolated double bond may be due to $\mathrm{C}=\mathrm{C}$ conjugation with the lone pair electrons of nitrogen in the molecule. The IR spectra of the final products $\mathbf{5}$ did not show the characteristic absorptions for $\mathrm{C}=\mathrm{O}$ and $\mathrm{NH}_{2}$ in the regions $1690-1650 \mathrm{~cm}^{-1}$ and $3445-3200 \mathrm{~cm}^{-1}$, respectively. On the other hand, a broadband in the region $3150-3140 \mathrm{~cm}^{-1}$ indicated the presence of a secondary amino group. This indicated that the reactions between 5-substituted-2-aminobenzenethiols and $\alpha, \beta$-unsaturated ketone had occurred in a concerted single step mechanism, without the isolation of any intermediate. The ${ }^{1} \mathrm{H}$ NMR showed a broad one proton absorption in the region 4.00-4.38 due to NH. In addition, the presence of two doublets, integrating for one proton each, at 6.60-6.95 and 7.25-7.46 support the formation of 2.5-dihydro derivatives, in preference to the 2,3-dihydro tautomer. The occurrence of the final products in the enamino-form is favored by the presence of $\mathrm{p}$ - conjugation (Scheme-1).

In the present communication, mainly covers structural elucidations of newly synthesized compounds done along with the brief description of the targets and we report piperidine mediated synthesis. The structures of the compounds 3a-b and $\mathbf{5 a - h}$ have been established on the basis of elemental $(\mathbf{C}, \mathrm{H}$, and $\mathrm{O})$ analysis, IR, ${ }^{1} \mathrm{H}$ NMR, MS spectral data and they were screened for Cytotoxic activity by using MTTmicro cultured (Tetrazolium)

\section{Cytotoxic Analysis}

Cellular viability in the presence of test compounds was determined by MTT-micro cultured. Tetrazolium assay. The cells seeded to flat bottom 96(10000cells/100ul) well plates \& cultured in the medium containing $10 \%$ serum and allowed to attach and recover for 24 hours in a humid chamber containing 5\% CO2. MTT (3-(4, 5-dimethylthiazol-2yl)-2,5diphenyl tetrazolium bromide; sigma catalog noM2128) was 
dissolved in PBS at $5 \mathrm{mg} / \mathrm{ml}$ and filtered to sterilize and remove a small amount of insoluble residue present in MTT. Different concentrations of compounds were added to the cells. After 48 hours, stock MTT solution (10ul) was added to the culture plate. Cells were again kept in CO2 incubator for 2 hours. After incubation 100ulof DMSO was added and mixed.
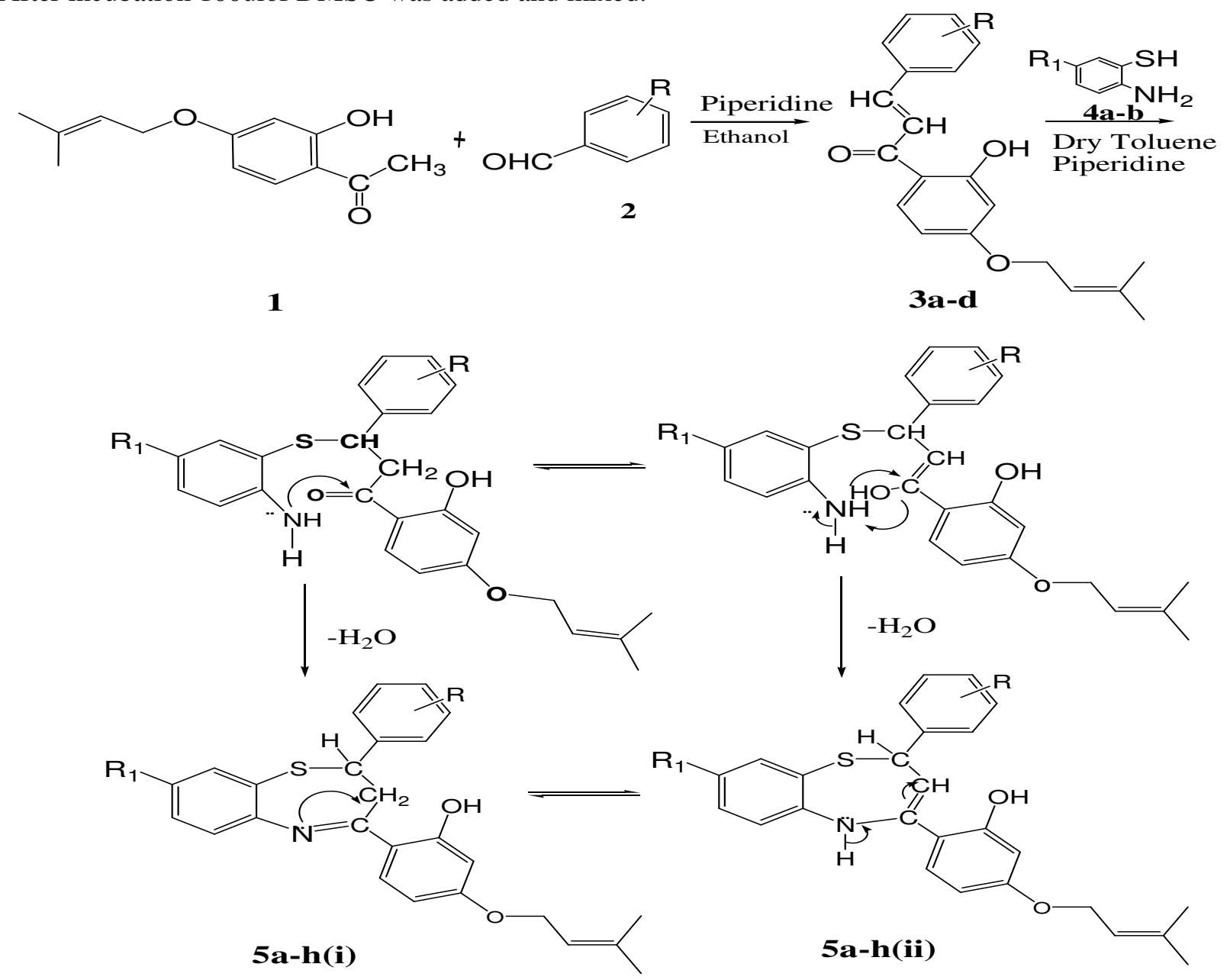

$\begin{array}{ccl}\text { Comp. } & \mathrm{R} & \text { Comp } \\ 3 \mathrm{a} & \mathrm{p}-\mathrm{OCH}_{3} & 4 \mathrm{a} \\ 3 \mathrm{~b} & \mathrm{p}-\mathrm{CH}_{3} & 4 \mathrm{~b} \\ 3 \mathrm{c} & \mathrm{p}-\mathrm{Cl} & \\ 3 \mathrm{e} & \mathrm{P}-\mathrm{N}\left(\mathrm{CH}_{3}\right)_{2} & \\ 3 \mathrm{f} & \mathrm{O}-\mathrm{Cl} & \end{array}$

$\begin{array}{llll}\mathrm{R}_{1} & \mathrm{Comp} & \mathrm{R} & \mathrm{R} \\ \mathrm{p}-\mathrm{CH}_{3} & 5 \mathrm{a} & \mathrm{p}-\mathrm{OCH}_{3} & \mathrm{p}-\mathrm{CH}_{3} \\ \mathrm{p}-\mathrm{OCH}_{3} & 5 \mathrm{~b} & \mathrm{p}-\mathrm{CH}_{3} & \mathrm{p}-\mathrm{OCH}_{3} \\ & 5 \mathrm{c} & \mathrm{p}-\mathrm{Cl} & \\ & 5 \mathrm{e} & \mathrm{p}-\mathrm{N}\left(\mathrm{CH}_{3}\right)_{2} & \\ & 5 \mathrm{f} & \mathrm{O}-\mathrm{Cl} & \end{array}$

Scheme-1

The absorbance was read at $562 \mathrm{~nm}$ in a plate reader. The results were represented as a percentage of cytotoxity/viability. All the experiments were carried out in triplicates. From the percentage of cytotoxicity the I C -50 value calculated.

Media used was MEM Catalog No M0643

DPBS Catalog No D5652

$1 \mathrm{X}$ antibiotic solution of 100X Catalog No A5955

1\% Sodium pyruvate Catalog No.S8636

$1 \%$ Non-essential amino acids Catalog No M7145

$10 \%$ Fetal bovine serum Catalog No F2442

DMSO Catalog No D5879

Trypsin-EDTA solution $(0.25 \%, 2.5$ g porcine trypsin and $0.2 \mathrm{~g}$ EDTA), Catalog NoT4049. 
Trypsin-EDTA solution used for detaching cells during sub culturing process. Cis-Platin was taken as reference.

Table-1: Cytotoxic Analysis

\begin{tabular}{c|c}
\hline Compound & IC50 Values in A-549 Cell Line \\
\hline $3 \mathrm{a}$ & $>100 \mathrm{mM}$ \\
\hline $3 \mathrm{~b}$ & $>100 \mathrm{mM}$ \\
\hline $3 \mathrm{c}$ & $>100 \mathrm{mM}$ \\
\hline $3 \mathrm{e}$ & $>100 \mathrm{mM}$ \\
\hline $3 \mathrm{f}$ & $15.05 \mathrm{uM}$ \\
\hline $5 \mathrm{a}$ & $14.54 \mathrm{uM}$ \\
\hline $5 \mathrm{~b}$ & $19.55 \mathrm{uM}$ \\
\hline $5 \mathrm{c}$ & $>100 \mathrm{mM}$ \\
\hline $5 \mathrm{~d}$ & $11.21 \mathrm{uM}$ \\
\hline $5 \mathrm{e}$ & $778.57 \mathrm{uM} 103.58 \mathrm{Um}$ \\
\hline $5 \mathrm{f}$ & $20.22 \mathrm{uM}$ \\
\hline $5 \mathrm{~g}$ & $>100 \mathrm{mM}$ \\
\hline $5 \mathrm{~h}$ & $>100 \mathrm{mM}$ \\
\hline
\end{tabular}

\section{ACKNOWLEDGMENT}

M. Sudha Rani \&N.B. thanks to the UGC minor project for the financial assistance and grateful to the $\mathrm{MD}$, GNITC, for supporting.

\section{REFERENCES}

1. H. Matsumoto, S. Hara, N. Nagata and K. Ikeda, Heterocyclic, 41, 47(1995), DOI:10.3987/COM-946871

2. H. Yamamoto, Y. Nakamura, Y. Kumoh, K. Ichihara, M. Nagasaka and H. Asai, Jpn. J. Pharmacol. Chem. Abstr., 41, 283(1986), DOI:10.1254/jjp.41.283

3. S. Ohno, K. Izumi, S. Mizukoshi, H. Yamamoto, M. Nagasaka and Y. Nakumara, J.P. Jpn. Kokai. Tokkyo. Kohochem. Abstr 6, 72,772 (86, 72,772) [Cl.C07 D281/10] ; 1986. 105 208946q.(1986)

4. Murako Pharmaceutical, Co. Ltd.; Jpn. Kokai. Tokkyo. Koho. JP; Chem. Abstr., 81,127,367(86, 72,772) [Cl.C07 D281/10] (1986), 96, 85601b(1981)

5. K. Itoh, M. Mor, Y. Inada, K. Nishikawa, V. Kawamatsu, \& H. Sugihara.; Chem. Pharm. Bull., 34, 3747(1986), DOI:10.1248/cpb.34.3747

6. D. M. Floyd and J. Krapcho, U.S. Patent, 4, 584, 131 (Cl.260-239,3B.C07 D281/10)1986; Chem. Abstr., 105, 78963x (1986).

7. O. Muras, T. Ikebe, I. Nakamata, K. Anami, and Jpn. Kokai. Tokyo. Koho. JP. Chem.abstr., 03,220,184 (91,220,184) [Cl.C07. D281/10], 116, 59416g.(1992)

8. R.A. Mane and D.B. Ingle, Indian. J. Chem., 21B, 973(1982), Chem. Abstr., 99, 22439w. (1983)

9. T. Yanamori, H. Harda, E. Oosugi, and K. Sakai, Eur. Pat. Appl. E.P 609,031(Cl.C07 C323/56), 1994, JP Appl. 93 / 11, 492; Chem. Abstr., 122, 10074d.(1995)

10. L. Yun., S. Na, and Sheng Jin. Chin., Chem. Lett., 10, 447(1999); Chem Abstr,131, 322450c.(1999)

11. L .Somogy, Synth., Commun. 29, 1857(1999), Chem. Abstr., 131, 58670h (1999).

12. Y. Li, J. Shi, Z. Dong, Y.J.J Sheng and Q.Y. Xing, Chin. Chem. Lett., 10, 23(1999), ChemAbstr, 131, 281800g. (1999)

13. J.F. Mais, R.H. Bloodworth, and B. Karsten, Von, Dem.; Ger, D. E. Offen, 19, 810, 392 (Cl.C07 C27/13) (1999), Appl. 19,810,392,1998. 10;Chem Abstr, 131, 199498v,(1999)

14. K. Waisser, L. Kubikova, J. Kaustova, H. Bartsch, T. Erker, and V. Hanus, Sci. Pharm., 67, 123(1999), Chem Abstr., 131, 226009v,(1999)

15. H. Christensen, E. Carlson,A. Asberg, L. Schram and K.J. Berg, Chin. Chim. Acta., 283, 63(1999), DOI: 10.1016/S0009-8981(99)00042-X

16. M. Amblard, I. Daffix, P. Bedos, G. Berge, D. Pruneau, J. Paquet, J.M. Luccarini, P. Belichard, P. Doddy and J Martinez, J. Med. Chem, 42, 4185(1999), DOI:10.1021/jm9901529 


\section{RASĀYAN J. Chem.}

Vol. 12 | No. 2 |796-802| April - June | 2019

17. N. Lapointe. H. Chen, D. Xu, S. Qi. P. Daloge and L. Dumount, Eur. Surg. Res, 31, 259(1999), Chem. Abstr. 131, 39484j(1999), DOI:10.1159/000008701

18. P. C. T. Eckmiller Marion, W.O. Int. Appl, 98, 50, 056 (Cl A 61 K38/55), D (1998) E. Appl., 19, 718, 826, 70pp (1997), Chem. Abstr. 130, 20593b(1999).

19. K.P. Yadav and D.B Ingle,. J. Indian Chem., 22B, 180(1983). Chem. Abstr. 99, 105221v(1983)

20. J.C. Muller, G. Lassalle and C. Denys, F. R. Fr-Demande, 2, 670, 785(1992) (Cl.C07 D417/12). Appl. 90 / 15, 988, 22(1990) pp. Chem. Abstr., 118, 124574(1993).

21. T. Yanamori, H. Harda, K. Sakai and K. Matasunaga, Eur. Pat. Appl. EP 541, 263 (Cl.C07 C281/10), (1993). J.P. Appl. 91 / 302, 348 (1991), Chem. Abstr,119, 180837k.(1993)

22. U.C. Pant and A. Bhatia. J. Indian. Het. Chem., 131, 6(1996).

23. U.C. Pant, Upreti Mani. Pant Seema, Dandia Anshu, Patnaik and A.K. Goyal. Phosphorus Sulphur and Silicon, 193, 126 (1997).

24. S. Pant, H. Chandra, P. Sharma and U.C. Pant, J. Pant. Indian. Chem., 45B, 1525 (2006)

25. U.C. Pant, A. Sharma, S. Pant and C.K. Sharma, Phosphorus. Sulphur and Silicon, 121, 117(1996).

26. A. Levai and H. Duddeck, Acta. Chim. Acad. Sci. Hung, 88, 293(1976).

27. H. Duddeck, M. Kaiser, and A. Levai, Leibig's. Ann. Chem, 869(1985), DOI: $10.1002 /$ jlac.198519850424

28. A. Levai, and H. Duddeck, Pharmazie, 38, 827(1983).

29. A. Levai, Pharmazie, 35, 680(1980).

30. C. H. Hankovszky, and K. Hideg, Acta. Chim. Acad. Sci. Hung., 68 , 403(1971)

31. U.C. Pant, H. Chandra, S. Goyal, A. Dandia and S. Pant, Phosphorus Sulpha Silicon and Releted Elements, 180-186(2005).

32. J.C. Vincent and H. Vincent, Proc. Exptl. Biol Med, 55, 162(1944).

[RJC-4078/2018] 